\title{
Inappropriate use of acid suppression therapy in Internal Medicine wards of a tertiary care teaching Hospital.
}

\author{
FaizanQaisar, *Ayesha Memon, Dileep Kumar, Muhammad AwaisMemon \\ ${ }^{I} R-4$ Resident M.D(Internal Medicine)Liquat University of Medical and Health Sciences Jamshoro,Sindh, \\ Pakistan \\ ${ }^{2,3,4}$ FCPS Part II TraineeLiquate University Of Medical And Health Sciences Jamshoro,
}

\begin{abstract}
Objective: To assess appropriateness of prescription of acid suppressive therapy (AST) in Internal Medicine ward in a tertiarycare hospital.

Methods: In this prospective observational study, weincluded all those patientswho were started on AST after admission in the internal medicine ward in a tertiary care hospital in Hyderabad, Sindh, Pakistan from May 1 to July 31, 2013. All those patientswere excluded from the study who were alreadyon the AST therapy. Details were taken regarding age, sex, primary diagnosis, other morbidity, drug used for AST and indication for AST on a specifically designed proforma. The appropriatenessof AST was determined by widely accepted Food and drug authority (FDA) indications and American society of health system pharmacist (ASHP) guidelines.

Results: We included300 Patients in our study. All patients, in our study, were given AST at the time of admission tothe medicine ward. 133 (44.33\%) Patients were male and 167 (55.66\%) were female, their mean age was $43.91 \pm 18.58$ (mean $\pm S D$ ). 53 (17.6\%) Patients had a FDA approved indication.51 (17\%) Patients received AST therapy for SUP (stress ulcer prophylaxis) Out of that 7 (2.3\%) had an absolute indication for SUP, 44(14.8\%) had 2 or more relative indications for SUP. 196 (65.3\%)received AST without any appropriate indication. Omeparzolewas themost frequently used AST (55\%), followed byranitidine (45\%) Conclusion:Use of AST is mostly inappropriate inInternal medicinewards.Implementation of institutional protocols, evidence based medicine teaching during residency training, and frequent review of therapy by the attending consultant during ward rounds may be helpfulto promote appropriate use of AST in medicine wards.

Keywords: Acid suppressive therapy, Liquate UniversityHospital, IUMHSJamshoro,Sindh, Pakistan
\end{abstract}

\section{Introduction:}

There are many FDA approved indications for the use of AST (acid suppressiontherapy) such as (Healing of erosive esophagitis Maintenance of healing of erosive esophagitis, Symptomatic gastro-esophageal reflux disease, Helicobacter pylori eradication in combination with antibiotics, Short-term treatment of active gastric, ulcer ,Short-term treatment of active duodenal ulcer,Maintenance of healed duodenal ulcer, Healing of NSAID-Associated gastric ulcer, Risk reduction of NSAID-associated gastric Ulcer, Pathologicalhypersecretory conditions including Zollinger-Ellison syndrome $)^{(1-2)}$ In addition, American society of health system pharmacist (ASHP) has also published,in 1983, the guidelines to administer AST for SUP (stress ulcer prophylaxis). ${ }^{(3)}$ Nevertheless, Several studies from all over the world, have reported that AST is prescribed inappropriately in hospitalized non-critically ill patients.In medical wards most prescriptions of AST are for stress ulcer prophylaxis or they are prescribed without alicensed indication. Inappropriately prescribed AST has potential for drug-drug interaction and agent specific side effects.Furthermore, AST can increase the risk of hospital acquired pneumonia and clostridium difficile infection. ${ }^{(4-15)}$ In Pakistan where poverty is a bigger problem, The cost of using drugs like PPIs,H2R blocker for an individual person, particularly for long-term is of utmost concern. ${ }^{(16)}$ In Pakistan, data on the appropriateness of AST in Medicine wards is available scarcely. Therefore,we conducted this study to see the appropriateness of acid suppressiontherapy inthe patientsadmitted to medical ward of Liaquat University Hospital,Hyderabad, Sindh, Pakistans.

\section{Material and Method:}

LiaquatUniversity Hospital is a tertiary care teaching hospital in public sector in Hyderabad, Sindh, Pakistan. There are 4 medicine wards in this hospital. Each ward has an assigned admission day. Cases from all four medicine wards were collected on their respective admission day. All thePatients who were prescribedAST after admission in the medical ward were included in the study. Patients, alreadyon AST, were excluded. Details were takenregardingage, sex, primary diagnosis, other morbidity, drug used for AST and indications for AST on a specifically designed Proforma. All the patients were categorized into 3 groups A, B, C respectively. Group A included the patients who were prescribed AST for stress ulcer prophylaxis. Group B included those who were started with AST for a FDA approved indications. Group C included the patients who were given AST without 
any licensed indication. We followed ASHP Guidelines to judge the appropriateness of AST for SUP.Prescription of AST for SUP was considered appropriate if the patient had 1 absolute indication i.e Coagulopathy (defined as platelet count $<50000 \mathrm{~mm}$ or an international normalization ratio of $>1.5$, or a partial thromboplastin time $>2$ times the control value, or requiring mechanical ventilation for $>48 \mathrm{~h}$ ), or 2 or more relative indications (Respiratory Failure ,Renal Failure, Heart Failure ,Hepatic dysfunction ,Jaundice ,Sepsis, Stroke ,Hypotension ,Previous Gastrointestinal Disease, High-Dose Corticosteroids $(>250 \mathrm{Mg}$ /Day Of Hydrocortisone), Thermal Injury To $>35 \%$, Heparin Or Warfarin, kidney or liver transplant, head injury). Treatment for Non-SUP was considered appropriate if patient had any of the FDA approved indications for AST that includes,(Healing of erosive esophagitis Maintenance of healing of erosive esophagitis, Symptomatic gastro esophageal reflux disease,Helicobacter pylori eradication in combination with antibiotics,Short-term treatment of active gastric, ulcerShort-term treatment of active duodenal ulcer, Maintenance of healed duodenal ulcer,Healing of NSAID-Associated gastric ulcer, Risk reduction of NSAIDassociated gastric Ulcer,Pathologicalhypersecretory conditions including Zollinger-Ellison syndrome) . AST was considered inappropriate in all those patients who had no FDA approved indications for AST as well as had no indication mentioned in ASHP guidelines for SUP.

\section{Results:}

We included 300 patients in our study.All patients in our study were given AST at the time of admission to Internal Medicine ward. $133(44.33 \%)$ were male and 167 (55.66\%) were female .Their mean age was $43.91 \pm 18.58$ (mean \pm SD). 53 (17.6\%) patients had a FDA approved indication (Symptomatic gastroesophageal reflux disease, Helicobacter pylori eradication in combination with antibiotics, Short-term treatment of active gastric ulcer, Risk reduction of NSAID-associated gastric Ulcer).51 (17\%) patient received AST therapy for SUP (stress ulcer prophylaxis) Out of that $7(2.3 \%)$ had an absolute indication for SUP, $\mathbf{4 4}(\mathbf{1 4 . 8 \%})$ had 2 or more relative indications for SUP . $196(\mathbf{6 5 . 3 \%})$ received AST without any appropriate indication. Omeparzolewas the most frequently used AST (55\%), followed by ranitidine $\mathbf{( 4 5 \% )}$

\section{Discussion:}

Currently, Alittle information is available on the prescription of acid suppression therapy in Pakistan. In our study we aimed to evaluate the appropriateness of prescription of AST in patients admitted to Internal Medicine ward.

Our study suggests that AST is commonly prescribed inappropriately in Internal Medicine ward. We included a total number of 300 patients in our study. All patients in our study were given AST at the time of admission to Internal Medicine ward. 133 (44.33\%) Patients were male and 167 (55.66\%) were female. their mean age was $43.91 \pm 18.58\left(\right.$ mean \pm SD). $53(17.6 \%)$ patients had a FDAapproved indications ${ }^{\text {(TablelII) }} .51(17 \%)$ Patients received AST therapy for SUP (stress ulcer prophylaxis) Out of that $7(2.3 \%)$ had an absolute indication for SUP, $44(14.8 \%)$ had 2 or more relative indications for SUP $\stackrel{(\text { Table IV })}{1} 196(65.3 \%)$ received AST without any appropriate indication $\stackrel{\text { (Table V) }}{-}$ Omeprazolewas themost frequently used AST (55\%), followed byRanitidine $(45 \%)$.

Our study agrees with the previous studies carried out on the same topic with slight variations within the results. In Italy gullotta et al carried out a single day survey of hospitalized patients at 20 centers and found that $27 \%$ received AST of which $51 \%$ was inappropriate ${ }^{(5)}$. A study conducted by Nardino et al in USA in a large community hospital reported that $54 \%$ of the hospitalized patients received AST of which $65 \%$ were inappropriate ${ }^{(4)}$. In addition,Parente et al reported the hospitalized patient receivingAST, $65.3 \%$ of the prescription wasinappropriate ${ }^{(7)}$. Furthermore Hwang et also conducted the similar study in noncriticallyhospitalized patients in a teaching hospital reported that $54.9 \%$ received AST of which $58.5 \%$ were inappropriate ${ }^{(10)}$ Treatment with AST in most of the patients who received AST unnecessarily was attributed to SUP. Although guidelines of ASHP are available to select the patient to administer AST for SUP and available medical literature also support indication of SUP only in ICU patientsNevertheless, practice of starting AST for SUP in non-critically ill patient admitted to medical ward has been increasing day by day with no scientific literature to support this practice. In our study we follow ASHP $\frac{\text { Table } 1}{1}$ to evaluate appropriateness of AST for SUP $^{(3)}$.One more important thing which was noticed, that in our study, almost every patient was started on AST by on-duty Postgraduate Fellow orResidentbut the next day after admission every treatment order was reviewed by aconsultant physician during morning ward round but no modification was made in the prescription order nor the AST was stopped.

The ASHP guidelinespublished, in 1989, do not include PPIs for SUP but in our study Omeprazole was the most common AST used for this purpose. (55\%). Othermedical sources also reported the PPIs are more commonlyprescribed drugs for SUP despite limited data is available to support this practice ${ }^{(17)}$.

AST is considered safe but current scientific data suggest that AST is associated with an increased risk of nosocomial pneumonia. ${ }^{(12)}$ AST can also increase risk of clostridium difficile infection. ${ }^{(13-15)}$ Furthermore AST 
has the potential for drug-drug interaction and agent specific side effects ${ }^{(3)}$ Financial implication of AST cannot be ignored in third world poverty-stricken country like Pakistan, Although ,in Pakistan, a little information is available on financial burden of AST but the annual cost of inappropriate SUP in Non -ICU patients were found to be nearly $\$ 111000$. $^{(8)}$

\section{Recommendations:}

The practice of prescribing AST unnecessarily can increase cost, drug interactions., and adverse events. It is of key importance in Pakistan where mostly patients are non-affording .Implementation of institutional protocols, evidence based medicine practice during residency training, and frequent review of therapy by the attending consultant during ward rounds may be helpful to promote appropriate use of AST in medicine ward.

American society of health system pharmacist Guidelines.

Table I

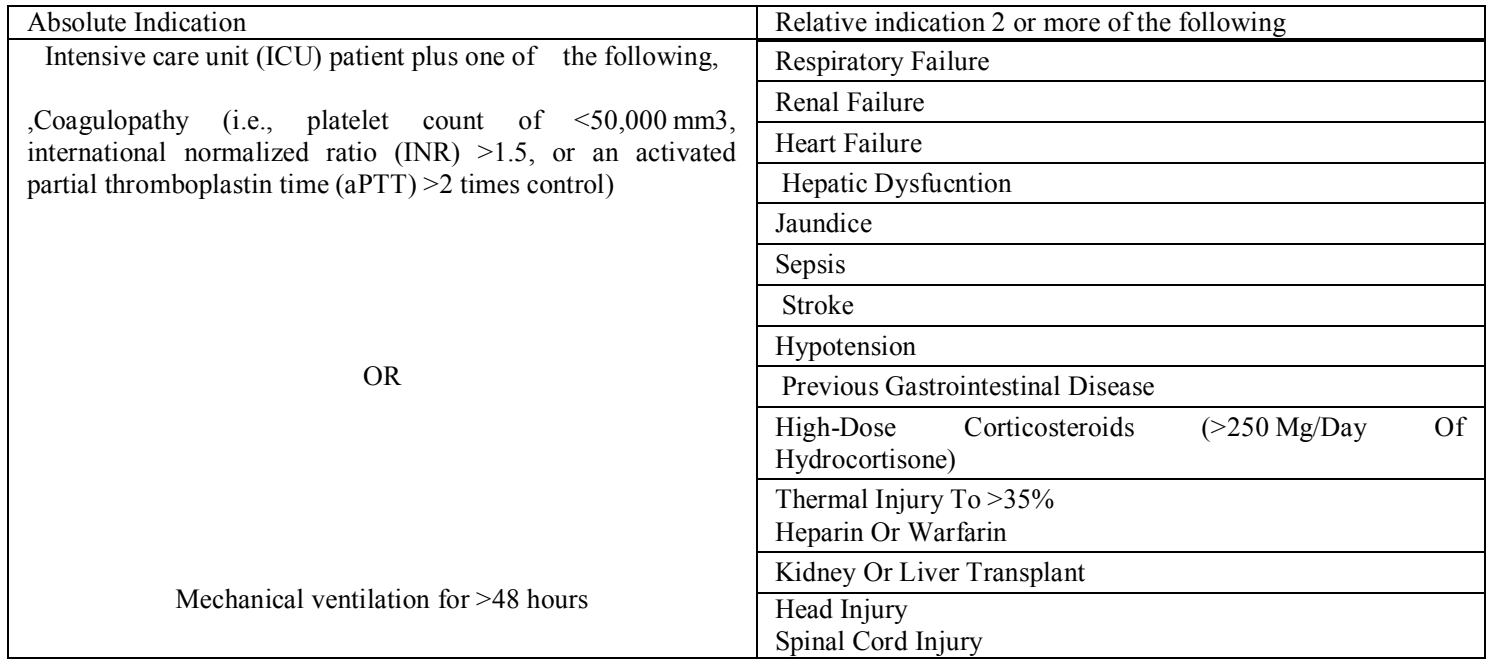

FDA approved indication for PPIs use.

Table II

\begin{tabular}{|ll|}
\hline$\bullet$ & Healing of erosive esophagitis \\
\hline$\bullet$ & Maintenance of healing of erosive esophagitis \\
\hline$\bullet$ & Symptomatic gastroesophageal reflux disease \\
\hline$\bullet$ & Helicobacter pylori eradication in combination with antibiotics \\
\hline$\bullet$ & Short-term treatment of active gastric ulcer \\
\hline$\bullet$ & Short-term treatment of active duodenal ulcer \\
\hline$\bullet$ & Maintenance of healed duodenal ulcer \\
\hline$\bullet$ & Healing of NSAID-Associated gastric ulcer \\
\hline$\bullet$ & Risk reduction of NSAID-associated gastric Ulcer \\
\hline$\bullet$ & Risk reduction of upper gastrointestinal bleeding in critically Ill patients \\
\hline$\bullet$ & Pathological hypersecretory conditions including Zollinger-Ellison syndrome \\
\hline
\end{tabular}

TableIII

FDA approved indication for AST in Group B Patients

\begin{tabular}{|c|l|c|c|}
\hline S.NO. & \multicolumn{1}{|c|}{ INDICATION } & No.of patients & $\%$ \\
\hline 1 & Helicobacter pylori eradication in combination with antibiotics & 03 & $1 \%$ \\
\hline 2 & Short-term treatment of active gastric ulcer & 6 & $2 \%$ \\
\hline 3 & Risk reduction of NSAID-associated gastric Ulcer & 09 & $3 \%$ \\
\hline 4 & Symptomatic gastroesophageal reflux disease & 35 & $11.66 \%$ \\
\hline
\end{tabular}

TableIV

AST used according to ASHP guidelines

\begin{tabular}{|l|c|c|}
\hline \multicolumn{1}{|c|}{ Indications } & No. of patients & Percentage \\
\hline Absolute indication & 7 & $2.33 \%$ \\
\hline Relative indication & 44 & $14.66 \%$ \\
\hline
\end{tabular}


Table V

Primary diagnosis of inappropriately prescribed AST Group.

\begin{tabular}{|l|l|c|c|}
\hline \multicolumn{1}{|c|}{ S.No } & \multicolumn{1}{|c|}{ Primary diagnosis } & No. of patients & $1 \%$ \\
\hline 1 & Unexplained fever & 3 & $1 \%$ \\
\hline 2 & Typhoid fever & 3 & $1 \%$ \\
\hline 3 & Diabetic ketoacidosis & 3 & $1 \%$ \\
\hline 4 & COPD & 3 & $1 \%$ \\
\hline 5 & Hepatic encephalopathy & 5 & $1.66 \%$ \\
\hline 6 & Organophosphorous poisoning & 5 & $1.66 \%$ \\
\hline 7 & Chronic kidney disease & 5 & $1.66 \%$ \\
\hline 8 & Malaria & 8 & $2.66 \%$ \\
\hline 9 & Uncontrolled diabetes & 8 & $2.66 \%$ \\
\hline 10 & Pulmonary T.B & 8 & $2.66 \%$ \\
\hline 11 & Meningitis & 13 & $4.33 \%$ \\
\hline 12 & Cerebral malaria & 13 & $4.33 \%$ \\
\hline 13 & Chronic liver disease & 33 & $11 \%$ \\
\hline 14 & Stroke & 37 & $12.33 \%$ \\
\hline 15 & Acute gastroenteritis & 46 & $15.33 \%$ \\
\hline 16 & Miscellaneous & & \\
\hline
\end{tabular}

\section{References:}

[1] Aciphex [package insert]. Eisai Inc. and Janssen Pharmaceuticals Inc., Titusville, NJ, USA, 2008

[2] Kapidex [package insert]. Takeda Pharmaceuticals America, Inc., Osaka, Japan, 2009

[3] ASHP therapeutic guidelines on stress ulcer prophylaxis: ASHP commission on therapeutics and approved by the ASHP Board of Directors on November 14, 1998. American Journal of Health-System Pharmacy. 1999;56:347-379

[4] Nardino RJ, Vender RJ, Herbert PN. Overuse of acid-suppressive therapyin hospitalized patients.Am J Gastroenterol. 2000; 95:31 $18-3122$.

[5] Gullotta R, Ferraris L, Cortelezzi C, Minoli G, Prada A, Comin U, Rocca F, Ferrara A, Curzio M. Are we correctlyusing the inhibitors of gastric acid secretion and cytoprotective drugs? Results of amulticentre study. Ital J GastroenterolHepatol. 1997;29:325-329.

[6] Pham CQ, Regal RE, Bostwick TR, Knauf KS. Acid suppressive therapyuse on an inpatient internal medicine service. Ann Pharmacother.2006;40:1 261-1 266.

[7] Parente F, Cucino C, G allus S, Bargiggia S, Greco S, Pastore L, Bianchi Porro G . Hospital use of acid-suppressive medications and its fall-out on prescribing in general practice: a 1 -month survey Aliment PharmacolTher. 2003; $17: 1$ 5031506.

[8] Heidelbaugh JJ, Inadomi JM. Magnitude and economic impact of inappropriate use of stress ulcer prophylaxis in non-I CU hospitalized patients. Am JGastroenterol. 2006; 101:2200-2205.

[9] Martín-Echevarría E, Pereira Juliá A, Torralba M, ArriolaPereda G, Martín Dávi la P, Mateos J, Rodríguez Zapata M. [Assessing the use of proton pump inhibitors in an internal medicine department] Rev EspEnferm Dig. 2008;100:76-8 1

[10] Hwang KO, Kolarov S, Cheng L, G riffith RA. Stress ulcer prophylaxis for non-critically ill patients on a teaching service. J EvalClin Pract.2007; 1 3:71 6-721.

[11] Forgacs I, Loganaygam A. Over prescribing proton pump inhibitor. BMJ. 2008; 336(7634):2-3.

[12] Herzig SJ, How ell MD, Ngo LH, Marcantonio ER. Acid-suppressive medication use and the risk for hospital-acquired pneumonia. JAMA.2009; $301: 2120-2128$.

[13] Cunningham R, Dale B, Undy B, G aunt N. Proton pump inhibitors as a risk factor for Clostridium difficilediarrhoea. J Hosp I nfect.2003; 54:243-245.

[14] Dial S, Alrasadi K, Manoukian C, Huang A, Menzies D. Risk of Clostridium difficile diarrhea among hospital inpatients prescribed proton pump inhibitors: cohort and case-control studies. CMAJ. 2004; $171: 33-38$.

[15] Louie TJ, Meddings J. Clostridium difficile infection in hospitals: risk factors and responses. CMAJ. 2004 ; $171: 45-46$.

[16] "World bank, UNDP question poverty estimates in Pakistan". OneWorld.net (South Asia). Retrieved 2008-05-04.

[17] Daley RJ, Rebuck JA, Welage LS, Rogers FB. Prevention of stress ulceration: current trends in critical care. Crit Care Med. 2004; 32:2008-2013.

Acknowledgment:

Dr.AbdulMajeedMemon, Dr.Muhammad Ali Memon ,Dr.FaizaMemon, Dr.RabiaMemon, Muhammad UmerMemon, Muhammad Hassan Memon. 